\title{
Preference reversals with losses
}

\author{
Daniel D. Holt, Leonard Green, Joel Myerson, and Sara J. Estle \\ Washington University, St. Louis, Missouri
}

\begin{abstract}
People who prefer larger, later gains over smaller, sooner gains when considering outcomes far in the future often reverse their preference as the alternatives become closer in time. This finding, which is contrary to a normative economic account of intertemporal choice, has been interpreted as support for hyperboloid discounting, but the results can also be explained by steeper discounting of smaller amounts. The present study is the first to demonstrate that analogous preference reversals occur with losses: People who preferred a smaller, sooner loss over a larger, later loss when the outcomes were far in the future reversed their preference when these alternatives were closer in time. Because there was no magnitude effect (i.e., smaller losses were not discounted more steeply than larger losses), the present findings strongly support the proposition that reversals in preference between delayed outcomes occur because of the hyperboloid shape of the discounting function.
\end{abstract}

Preference reversals are a common occurrence in everyday decision making. For example, a person may decide in the morning to eat a healthy dinner that evening but then pick up fast food (with fries) on the way home. Such behavior is obviously relevant to issues of applied significance like self-control and impulsivity. Moreover, such behavior has important theoretical implications, which stem from the fact that although preference reversals are an anomaly from the viewpoint of standard economic theory (Loewenstein, 1992), they can be understood in the context of the delay-discounting framework (Green \& Myerson, 2004).

According to the discounting interpretation, the value of a delayed outcome decreases as a function of the increase in the time until the expected occurrence of that outcome, following a hyperboloid (i.e., hyperbola-like) trajectory. That is, the present value, $V$, of a delayed outcome is given by

$$
V=\frac{A}{(1+k D)^{s}},
$$

where $A$ represents the amount of the outcome, $D$ is the delay, the parameter $k$ governs the rate of discounting, and the parameter $s$ reflects the nonlinear scaling of amount and time (Myerson \& Green, 1995). Note that when $s=1$, Equation 1 reduces to a simple hyperbola (Mazur, 1987).

Hyperboloid discounting predicts that even though one may prefer a larger, later reward (e.g., a nutritious dinner with positive health consequences) over a smaller, sooner reward (e.g., burger and fries on the way home) when both are relatively far in the future, one's preference may reverse as the time for the evening meal approaches. Such a reversal in preference is depicted in the top half of Figure 1, in which the heights of the bars represent what the values of two rewards would be if they were received immediately and the curves describe the hyperboloid decrease in their values as the delays until their receipt increase. Note that an individual making a choice at $T_{A}$, far in advance of either outcome, would prefer the larger, later reward whose present value (represented by the solid curve) is greater than that of the smaller, sooner reward (represented by the dashed curve). As time passes and the delays shorten, however, the two discounting curves cross, and the present value of the smaller, sooner reward becomes greater than that of the larger, later reward. Thus, the same individual choosing at $T_{B}$, close in time to the two outcomes, would now prefer the smaller, sooner reward.

Hyperboloid discounting also predicts preference reversals with delayed losses. The reversals predicted for losses, however, are opposite in direction to those observed with delayed gains, because with losses, a smaller loss has greater value than a larger loss. As depicted in the bottom half of Figure 1, if the choice is made at $T_{A}$, well in advance of the two outcomes, the smaller, sooner loss would be preferred because it has a higher present value (i.e., its value is less negative). As the delays get shorter, however, the discounting curves cross, and thus at $T_{B}$, closer in time to the two outcomes, the larger, later loss is the less negatively valued of the two and would be preferred.

Although it is well established that preference reversals occur when the delayed outcomes are gains (Green, Fristoe, \& Myerson, 1994; Kirby \& Herrnstein, 1995; Millar \& Navarick, 1984; Solnick, Kannenberg, Eckerman, \& Waller, 1980), preference reversals with delayed losses have not been studied, even though credit cards have made such reversals ubiquitous. For example, at the beginning of the month, an individual attempting to manage his or her household debt may prefer to pay credit card bills in full rather than deferring part of the payment and incurring additional charges. At the end of the month, however, when the bills actually

L. Green, lgreen@wustl.edu 


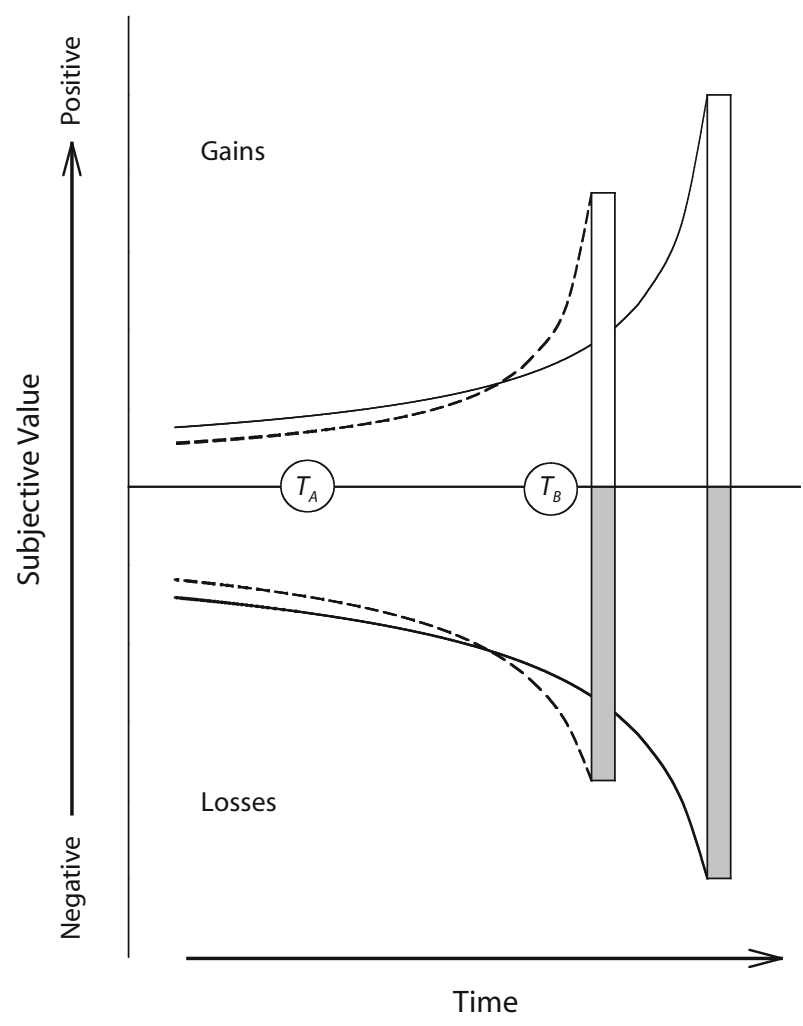

Figure 1. Preference reversals with gains and losses. The upper half of the figure depicts hypothetical data for delayed gains, and the lower half depicts hypothetical data for delayed losses. The heights of the white bars represent the subjective values of a smaller, sooner reward and a larger, later reward if these rewards were to be received immediately, and the heights of the dark bars represent the subjective values of a smaller, sooner payment and a larger, later payment if these payments had to be made immediately. The curves represent the subjective values of the outcomes at different points in time, assuming hyperboloid discounting. Note that for the gains, the subjective value of the larger, later outcome is greater (more positive) than that of the smaller, sooner outcome at $T_{A}$, when both outcomes are relatively far in the future, whereas for the losses, the subjective value of the smaller, sooner outcome is greater (less negative) at $\boldsymbol{T}_{A}$. As time passes, however, the relative subjective values of the two outcomes reverse so that at $\boldsymbol{T}_{B}$, when both outcomes are relatively close in time, the subjective values of the smaller, sooner gain and the larger, later loss are now the ones with the greater subjective values.

have to be paid, the individual may end up making only the minimum payment. Although this example makes intuitive sense, there is no experimental evidence that humans actually behave this way or, if they do, that their behavior can be modeled using hyperboloid discounting functions.

In order to determine whether preferences involving delayed losses actually reverse, we employed a procedure modeled after that used by Green et al. (1994). We began the procedure by adjusting the difference in the delays for a smaller and a larger payment until subjects preferred the option of paying the larger amount of hypothetical money later rather than the option of having to pay a smaller amount sooner. We then added equal amounts of time to both delays to see if preferences would reverse.
In addition to determining whether preference reversals occurred with delayed losses, the present experiment examined the effect of amount of payment on preference. Green et al. (1994), in their study of preference reversals with delayed gains, varied the absolute amounts of the smaller, sooner and larger, later rewards while holding the ratio of the two amounts constant. They reported that as the absolute amounts increased, less time needed to be added to the delays before preference reversed. This finding is consistent with the magnitude effect observed with delayed rewards, in which larger rewards are discounted less steeply than smaller rewards (see, e.g., Green, Myerson, \& McFadden, 1997; Kirby, 1997). However, the magnitude effect by itself can explain preference reversals without appealing to the form of the discounting function. For example, even if discounting functions were exponential, as is frequently assumed by economists, steeper discounting of the smaller amount would give rise to preference reversals, whereas in the absence of such a magnitude effect, exponential discounting functions would not predict preference reversals (Green \& Myerson, 1993).

In a recent study (Estle, Green, Myerson, \& Holt, 2006; see also Ostaszewski \& Karzel, 2002) comparing the discounting of delayed losses with the discounting of delayed gains, we found that the magnitude effect observed with losses was smaller and less reliable (if it occurred at all) than the magnitude effect observed with delayed gains. To assess the generality of this unexpected finding, we varied the absolute amounts of the smaller, sooner payments and the larger, later payments, while holding their ratio constant, in order to evaluate the effect of amount on choice between delayed losses. If there were no magnitude effect with losses but preference reversals were still observed, this would provide strong support for the proposition that preference reversals between delayed outcomes occur because of the hyperboloid shape of the discounting function.

\section{METHOD}

\section{Participants}

The participants were 46 undergraduate students, 19 males and 27 females, recruited from the human subjects pool maintained by the Department of Psychology at Washington University in St. Louis.

\section{Procedure}

Participants were tested individually in a small testing room. The experimenter controlled a series of PowerPoint slides that were presented on a computer monitor. The experimenter and participant were seated so that their views of each other were obscured by their respective monitors. The slide for each trial showed a pair of hypothetical amounts of money - a smaller amount to be paid sooner and a larger amount to be paid later - and participants said whether they would prefer to make the smaller, sooner payment or the larger, later payment.

The experiment was a 4 (amount-pair) $\times 11$ (delay-difference) factorial within-subjects design, where delay-difference is defined as the delay until the later payment minus the delay until the sooner payment. In each of the four amount-pair conditions, the smaller, sooner payment was two thirds the size of the larger, later payment: $\$ 80$ in the sooner condition versus $\$ 120$ in the later; $\$ 500$ versus $\$ 750 ; \$ 2,000$ versus $\$ 3,000$; and $\$ 12,000$ versus $\$ 18,000$. The 11 delay-difference conditions involved differences of 1 week, 1 month, 3 months, 6 months, 
1 year, 3 years, 5 years, 7 years, 10 years, 15 years, and 20 years between the sooner and the later payment options.

For each combination of amount-pair and delay-difference, there were also 16 possible delays until the smaller amount was to be paid: immediately, or after a delay of 1 week, 2 weeks, 1 month, 3 months, 6 months, 1 year, 2 years, 3 years, 5 years, 7 years, 10 years, 12 years, 15 years, 17 years, or 20 years. For each participant in each amountpair condition, we first determined the smallest delay-difference at which the participant chose the larger, later payment over an immediate, smaller payment. This was accomplished by increasing the difference between the delays until the larger payment was preferred.

Once the participant indicated a preference for the larger payment, the delay to the sooner payment was increased (while the difference between the delays was held constant) until the participant's preference reversed and the smaller, sooner payment was preferred or until the longest (20-year) delay was reached, whichever happened first. Then the next longest delay-difference was studied, again beginning with an immediate, smaller payment and increasing the delay to the sooner payment until the smaller payment was preferred (or the longest delay was reached). This procedure was repeated until the participant had been studied in this amount-pair condition at each delay-difference. The order in which the four amount-pair conditions were presented was randomly selected for each participant.

At the beginning of the session, participants received the following instructions:

In the following task you will be presented with a series of hypothetical amounts of money to be paid at different times. Your job is simply to decide whether you would rather pay the smaller amount of money sooner or the larger amount of money later. You might imagine situations in which you are assessed a fine for a parking violation, for example, and you can either pay the fine sooner in time, say $\$ 60$ now, or you can pay it later but with an additional cost for delay of payment, $\$ 90$ dollars. Similarly, perhaps you hire a contractor to do work on your house and he gives you the option of paying $\$ 1,200$ in cash up front, or mailing him a check later for $\$ 1,400$ dollars. As you think of these types of payment situations and indicate your own choice, remember that there are no right or wrong responses; we only ask that you give your honest and thoughtful preference.

Participants then performed a brief series of practice trials and, if there were no questions, the experimental trials began.

\section{RESULTS}

Of fundamental interest in the present experiment was whether preference reversals like those observed when the outcomes are gains would also be observed when the outcomes are losses. The answer to this question is yes. Participants who preferred a larger, later payment when the time until the smaller payment was brief typically reversed their preference and came to choose the smaller payment over the larger, later payment as the delays until both payments increased. For example, when given a choice between making an immediate $\$ 2,000$ payment or a $\$ 3,000$ payment in 3 years, three fourths of the participants preferred to make the larger payment; all of these participants, however, reversed their preference as the delays until both the smaller and larger payments increased.

Given that preference reversals occur, we were interested in the factors that determine when they occur. That is, we wanted to know how participants' preferences for paying a small amount sooner rather than a larger amount later would be affected by (1) the delay until the sooner payment, (2) the difference in time between when the sooner payment would have to be made and when the later one would have to be made (i.e., the delay-difference), and (3) the absolute size of the amounts involved (i.e., the amount-pair). In order to answer these questions, we determined for each participant in each delay-difference and amount-pair condition how long the delay until the smaller, sooner payment had to be before such a payment was preferred over the larger, later payment. We then submitted these delays to a 4 (amount-pair) $\times 11$ (delay-difference) repeated measures ANOVA. There was a significant effect of delay-difference $[F(10,440)=27.72, p<.001]$. A significant linear contrast $[F(1,213)=32.79, p<.001]$ revealed that this effect reflected the fact that as the delaydifference increased, an increasingly larger delay to the smaller payment was required before it was preferred over the larger, later payment. There was no effect of the absolute size of the amount-pair, whether assessed as a main effect or by testing for a linear contrast (both $F \mathrm{~s}<1.10$ ), and there was no significant interaction between delaydifference and amount-pair $(F<1.0)$.

Figure 2 plots the proportion of participants choosing the smaller, sooner payment as a function of the delay until the sooner payment. Because of the large number of conditions involved (44 combinations of amount-pairs and delay-differences), only the data from every other delay-difference condition are shown, with the selected delay-difference conditions shown in separate panels, beginning with the smallest difference (1 week) and ending with the largest (20 years).

As may be seen, the proportion of participants choosing the smaller, sooner payment increased as the weeks until the sooner payment increased. This occurred in each delaydifference condition and, as can be seen, was not systematically affected by the absolute amounts of the payments. In contrast, the size of the difference between the two delays systematically affected both (1) the proportion of participants choosing a sooner payment when that payment was immediate and (2) the rate at which the proportion choosing the sooner payment increased as a function of the delay until that payment. More specifically, the proportion choosing an immediate, smaller payment was highest in the smallest (1-week) delay-difference condition, and it decreased steadily as the delay-difference increased (compare the leftmost data points, which correspond to immediate, smaller payments, in the various panels). Moreover, the rate of increase in the proportion choosing the sooner payment as the delay until that payment increased was higher for smaller delay-differences and lower for larger delaydifferences (compare the curves in the various panels).

To verify that the results reflected systematic changes in preference as a function of increases in the delay until the sooner payment rather than chance fluctuations, we plotted the proportion still preferring the larger, later payment from each delay-difference condition (i.e., 1 minus the proportion shown in Figure 2) as a function of the number of previous choices in that condition. Rather than the exponential decay predicted by chance fluctuations, the 


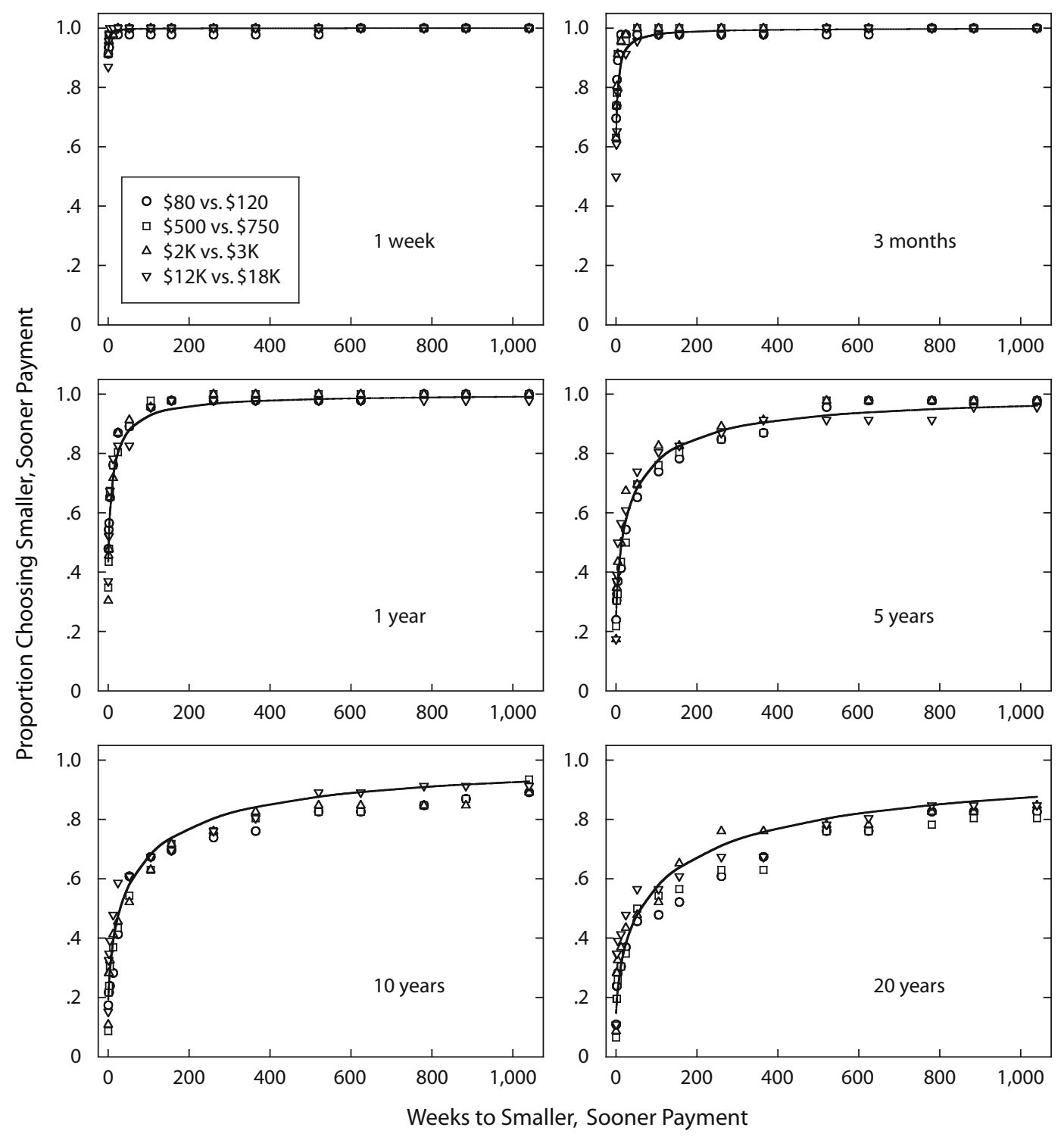

Figure 2. Proportion of participants choosing the smaller, sooner payment as a function of the time until that payment would have to be made. Each panel depicts data from a specified delay-difference condition (indicated in the lower right corner of the panel); data from the four amount-pair conditions are represented by different symbols. The curves represent the best-fitting group preferences function (Equation 2) fit to the data from all 11 delay-difference conditions simultaneously. Because of the large number of conditions studied, only data from every other delay-difference condition are shown.

resulting survival functions showed approximately linear decreases, thereby demonstrating that chance fluctuations were not responsible for the observed pattern of results.

The pattern of results shown in Figure 2 is captured by the equation

$$
P_{\mathrm{S}}=\frac{1+\frac{A_{\mathrm{S}}}{A_{\mathrm{L}}}}{1+\frac{A_{\mathrm{S}}}{A_{\mathrm{L}}}\left(1+\frac{k \Delta}{1+k D}\right)^{s}},
$$

where the proportion $\left(P_{\mathrm{S}}\right)$ of participants choosing the smaller, sooner payment is a function of the delay until the sooner payment $(D)$ and the delay-difference $(\Delta)$, as well as the ratio of the amounts of the sooner and later payments $\left(A_{\mathrm{S}}\right.$ and $\left.A_{\mathrm{L}}\right)$. As in Equation 1, the $k$ parameter in the group preferences function (Equation 2) reflects the rate of discounting (a larger $k$ indicates steeper discounting), and the $s$ parameter reflects the nonlinear scaling of amount and time (the extent to which $s$ deviates from 1.0 indicates the degree of nonlinearity). The curved lines in Figure 2 correspond to Equation 2 fit to all of the data simultaneously using two independent variables ( $D$ and $\Delta$ ) and only two free parameters $(k$ and $s)$, with $A_{\mathrm{S}}: A_{\mathrm{L}}$ held constant at $2: 3$, the ratio of the smaller and larger payments in all of the amount-pair conditions.

Equation 2 follows from three assumptions (see the Appendix for derivation). First, the present value of a delayed outcome is a hyperboloid function of delay (Equation 1; 
Green et al., 1994; Myerson \& Green, 1995; for a review, see Green \& Myerson, 2004). Second, preference depends on the relative present value of the two alternatives, $V_{\mathrm{L}} /\left(V_{\mathrm{S}}+V_{\mathrm{L}}\right)$ (Luce, 1959; Rachlin, 1989). Recall that because the choices involve payments (rather than rewards), a payment with a smaller (i.e., less negative) present value is preferred over a payment with a larger (i.e., more negative) present value. Thus, the proportion choosing the smaller, sooner payment will increase as the relative present value of the larger, later payment increases. Finally, the proportion choosing the sooner payment is assumed to reach asymptote at 1.0.

Equation 2 predicts that increasing the delay $(D)$ until the smaller, sooner payment will increase the proportion choosing that option, and the data are consistent with this prediction (as can be seen in each of the panels of Figure 2). Equation 2 also predicts that increasing the difference between the delays $(\Delta)$ until the two payments will decrease the proportion choosing the smaller payment, and the data are consistent with this prediction as well (as can be seen by looking across the panels of Figure 2). When fit to the data from all of the amount-pair and delaydifference conditions, the group preferences function (i.e., Equation 2) with $k=0.509(S E=0.035)$ and $s=0.436$ $(S E=0.004)$ accounted for $97.0 \%$ of the variance.

\section{DISCUSSION}

Previous studies have demonstrated that when people choose between delayed gains, one smaller but available sooner and the other larger but available later, they often prefer the larger amount even though it occurs later, at least when both outcomes are far in the future. When choosing between delayed gains that are closer in time, however, people often reverse their preference and choose the smaller, sooner option (Green et al., 1994; Kirby \& Herrnstein, 1995; Millar \& Navarick, 1984; Solnick et al., 1980). The present results provide the first demonstration that preference reversals also occur when people choose between two delayed losses. In this case, however, people often preferred a smaller, sooner payment when both hypothetical outcomes were far in the future, but preferred the larger, later payment when these outcomes were closer to the present.

Consistent with the view that delayed outcomes are discounted in a hyperboloid fashion and that preference reflects the relative present value of the outcomes, the proportion of participants choosing the smaller, sooner payment increased with increases in the delay until the sooner payment and decreased with increases in the difference between the delay until the smaller, sooner outcome and the larger, later outcome. However, the present results revealed no evidence that the absolute amounts of the sooner and later payments affect preference so long as the ratio of the two amounts is held constant, as was the case in the present experiment. This finding is consistent with our previous finding that magnitude effects, if they occur at all with losses, are much smaller and less reliable than the magnitude effects that occur when the delayed outcomes are gains (Estle et al., 2006; see also Ostaszewski \& Karzel, 2002).
The fact that preference reversals were observed in the absence of a magnitude effect has important theoretical implications. The occurrence of preference reversals with delayed gains has often been cited as evidence that discounting functions are hyperboloid rather than exponential (see, e.g., Ainslie, 1992, 2001). As Green and Myerson (1993) pointed out, however, in principle, such reversals could occur if discounting were exponential, given that smaller rewards are discounted more steeply than larger rewards. The occurrence of preference reversals with losses in the absence of a magnitude effect in the present study argues against this alternative explanation for preference reversals and provides strong support for hyperboloid discounting.

Our group preferences function (Equation 2) captured the pattern of choices between delayed losses at the group level, accounting for $97 \%$ of the variance in 704 data points from 44 conditions using only two parameters, corresponding to the $k$ and $s$ parameters of the discounting function (Equation 1). The group preferences function instantiates two key theoretical assumptions regarding the process of choosing between delayed losses: first, that individual discounting functions are hyperboloid (Equation 1), and second, that preference is based on relative present value. The accuracy with which Equation 2 parsimoniously described the results is consistent with these assumptions. Moreover, the fact that it was not necessary to assume different $k \mathrm{~s}$ for different amount-pair conditions in order to describe the present data is supportive of Estle et al.'s (2006) contention that magnitude effects with losses tend to be small and unreliable.

It should be noted that the group preferences function is not intended to represent the average of the functions describing the preferences of individual participants. Indeed, inferring the form of functions at the individual level from the form at the aggregate level is fraught with problems (Anderson \& Tweney, 1997; Sidman, 1952; Wixted \& Ebbesen, 1997). Although the form of the discounting function that describes individuals' choices between an immediate and a delayed loss is now known to be hyperboloid (Estle et al., 2006), verifying that the function has that same form when individuals choose between two delayed losses, as in the present study, will require research that directly addresses this issue.

We would stress, however, that the group preferences function does instantiate principles that appear to govern individual preferences (i.e., hyperboloid discounting and matching of relative value) and thus makes it possible to interpret aggregate behavior in terms of these principles. For example, under circumstances that lead to steeper discounting of losses (i.e., higher values of $k$ ), there will be a wider time window (i.e., a greater range of times until the sooner, smaller payment) within which people choose to make a larger, later payment. This parallels what one would expect to observe at the individual level: An individual who tends to steeply discount the impact of delayed losses (i.e., someone with a high value of $k$ ) may state a preference for making a smaller, sooner payment when the payment is far in the future; as the time to actually make the payment approaches, however, he or she will tend to undergo a prefer- 
ence reversal sooner (and decide to postpone making the payment even if doing so means making a larger payment later) than will someone who discounts delayed losses less steeply. The group preferences function may prove useful in economic applications, in which the fact that this function instantiates principles governing individual behavior would be advantageous, since such applications often rely on microeconomic principles for their interpretation.

Many situations in everyday life require us to choose between delayed outcomes, and the choices we make when these outcomes are far in the future may be contrary to what we prefer when the same options are reconsidered at a later time. If we committed to a particular option and are thus locked in, then subsequent preference reversals may be associated with regret. Alternatively, if we are not locked in, then there is the possibility that we will act on our reversed preference. In the latter case, our behavior may be characterized as impulsive or indicative of a lack of self-control. Despite its obvious implications for issues of applied significance like self-control and impulsivity, choice between delayed losses has received little attention. The present study sheds light on how the timing of events (e.g., when the choice is made relative to when its later consequences will occur) controls preference in these situations and allows choice between delayed losses to be integrated within a larger discounting framework.

\section{AUTHOR NOTE}

This research was supported by Grant R01 MH055308 from the National Institute of Mental Health. D.D.H. is now at the University of Wisconsin-Eau Claire. We thank Jake Ackerman for his assistance with data collection. Correspondence concerning this article should be addressed to L. Green, Department of Psychology, Washington University, St. Louis, MO 63130 (e-mail: lgreen@wustl.edu).

\section{REFERENCES}

Ainslie, G. (1992). Picoeconomics: The strategic interaction of successive motivational states within the person. Cambridge: Cambridge University Press.

Ainslie, G. (2001). Breakdown of will. Cambridge: Cambridge University Press.

Anderson, R. B., \& Tweney, R. D. (1997). Artifactual power curves in forgetting. Memory \& Cognition, 25, 724-730.
Estle, S. J., Green, L., Myerson, J., \& Holt, D. D. (2006). Differential effects of amount on temporal and probability discounting of gains and losses. Memory \& Cognition, 34, 914-928.

Green, L., Fristoe, N., \& Myerson, J. (1994). Temporal discounting and preference reversals in choice between delayed outcomes. Psychonomic Bulletin \& Review, 1, 383-389.

Green, L., \& Myerson, J. (1993). Alternative frameworks for the analysis of self control. Behavior \& Philosophy, 21, 37-47.

Green, L., \& Myerson, J. (2004). A discounting framework for choice with delayed and probabilistic rewards. Psychological Bulletin, 130, 769-792.

Green, L., Myerson, J., \& McFadden, E. (1997). Rate of temporal discounting decreases with amount of reward. Memory \& Cognition, 25, 715-723.

KIRBY, K. N. (1997). Bidding on the future: Evidence against normative discounting of delayed rewards. Journal of Experimental Psychology: General, 126, 54-70.

Kirby, K. N., \& Herrnstein, R. J. (1995). Preference reversals due to myopic discounting of delayed reward. Psychological Science, 6, 83-89.

Loewenstein, G. (1992). The fall and rise of psychological explanations in the economics of intertemporal choice. In G. Loewenstein \& J. Elster (Eds.), Choice over time (pp. 3-34). New York: Russell Sage Foundation.

LuCE, R. D. (1959). Individual choice behavior: A theoretical analysis. New York: Wiley.

MAZUr, J. E. (1987). An adjusting procedure for studying delayed reinforcement. In M. L. Commons, J. E. Mazur, J. A. Nevin, \& H. Rachlin (Eds.), Quantitative analyses of behavior: Vol. 5. The effect of delay and of intervening events on reinforcement value (pp. 55-73). Hillsdale, NJ: Erlbaum.

Millar, A., \& Navarick, D. J. (1984). Self-control and choice in humans: Effects of video game playing as a positive reinforcer. Learning \& Motivation, 15, 203-218.

Myerson, J., \& Green, L. (1995). Discounting of delayed rewards: Models of individual choice. Journal of the Experimental Analysis of Behavior, 64, 263-276.

Ostaszewski, P., \& Karzel, K. (2002). Discounting of delayed and probabilistic losses of different amounts. European Psychologist, 7, 295-301.

Rachlin, H. (1989). Judgment, decision, and choice: A cognitivel behavioral synthesis. New York: Freeman.

Sidman, M. (1952). A note on functional relations obtained from group data. Psychological Bulletin, 49, 263-269.

Solnick, J. V., Kannenberg, C., Eckerman, D. A., \& Waller, M. B. (1980). An experimental analysis of impulsivity and impulse control in humans. Learning \& Motivation, 11, 61-77.

Wixted, J. T., \& EbBesen, E. B. (1997). Genuine power curves in forgetting: A quantitative analysis of individual subject forgetting functions. Memory \& Cognition, 25, 731-739. 


\section{APPENDIX}

The group preferences function (Equation 2) follows from three assumptions. The first of these assumptions is that the present value of a delayed outcome is assumed to be a hyperboloid function of delay. Thus, the present value of the smaller, sooner outcome is given by $V_{\mathrm{S}}=A_{\mathrm{S}} /(1+k D)^{s}$, and the present value of the larger, later outcome is given by $V_{\mathrm{L}}=A_{\mathrm{L}} /[1+k(D+\Delta)]^{s}$, where $A_{\mathrm{S}}$ and $A_{\mathrm{L}}$ represent the smaller and larger amounts, respectively, $D$ is the delay until the sooner amount, and $\Delta$ is the difference between the delay until the smaller, sooner outcome and the delay until larger, later outcome. Note that the equation for the present value of the larger, later outcome may be rewritten as $V_{\mathrm{L}}=A_{\mathrm{L}} /(1+k D+k \Delta)^{s}$.

The second assumption is that the proportion of individuals choosing the smaller, sooner payment, $P_{\mathrm{S}}$, depends on the relative present value of the larger, later outcome:

$$
P_{\mathrm{S}}=c\left[\frac{V_{\mathrm{L}}}{V_{\mathrm{L}}+V_{\mathrm{S}}}\right] \text {. }
$$

Given hyperboloid discounting (our first assumption), relative present value may be written as

$$
\frac{V_{\mathrm{L}}}{V_{\mathrm{L}}+V_{\mathrm{S}}}=\frac{\frac{A_{\mathrm{L}}}{(1+k D+k \Delta)^{s}}}{\frac{A_{\mathrm{L}}}{(1+k D+k \Delta)^{s}}+\frac{A_{\mathrm{S}}}{(1+k D)^{s}}} .
$$

It should be noted that because we are dealing with losses, a smaller negative value is preferred over a larger one, and thus a larger relative present value will be associated with preference for the smaller, sooner payment.

Dividing both the numerator and the denominator of the expression on the right in Equation $\mathrm{A} 2$ by $A_{\mathrm{L}} /(1+$ $k D+k \Delta)^{s}$ yields

$$
\frac{V_{\mathrm{L}}}{V_{\mathrm{L}}+V_{\mathrm{S}}}=\frac{1}{1+\frac{\frac{A_{\mathrm{S}}}{\frac{(1+k D)^{s}}{(1+k D+k \Delta)^{s}}}}{\left(1+\frac{A_{\mathrm{L}}}{(1+k)}\right.}} .
$$

Simplifying the fraction in the denominator of the expression on the right, one may obtain

$$
\frac{V_{\mathrm{L}}}{V_{\mathrm{L}}+V_{\mathrm{S}}}=\frac{1}{1+\frac{A_{\mathrm{S}}}{A_{\mathrm{L}}}\left(\frac{1+k D+k \Delta}{1+k D}\right)^{s}},
$$

which may be simplified further, yielding

$$
\frac{V_{\mathrm{L}}}{V_{\mathrm{L}}+V_{\mathrm{S}}}=\frac{1}{1+\frac{A_{\mathrm{S}}}{A_{\mathrm{L}}}\left(1+\frac{k \Delta}{1+k D}\right)^{s}} .
$$

The third and final assumption is that the proportion of individuals choosing the sooner payment, $P$, increases as a function of the delay until the sooner payment and reaches asymptote at 1.0. Inspection of the immediately preceding equation for relative present value, however, reveals that as $D$ increases, the relative value of the larger, later outcome approaches $1 /\left(1+A_{\mathrm{S}} / A_{\mathrm{L}}\right)$. Therefore, it follows from the third assumption that the parameter $c$ in Equation $\mathrm{A} 1$ is equal to $\left(1+A_{\mathrm{S}} / A_{\mathrm{L}}\right)$, and thus, the proportion of individuals choosing the smaller, sooner payment is given by the group preferences function specified in Equation 2. 